Check for updates

Cite this: RSC Adv., 2019, 9, 1106

Received 14th November 2018 Accepted 20th December 2018

DOI: $10.1039 / c 8 r a 09386 e$

rsc.li/rsc-advances

\section{Amino-functionalized magnetic chitosan beads to enhance immobilization of potassium copper hexacyanoferrate for selective $\mathrm{Cs}^{+}$removal and facile recovery $\dagger$}

\begin{abstract}
Hyelin Roh, ${ }^{a}$ Yonghwan Kim, ${ }^{a}$ Yun Kon Kim, ${ }^{a}$ David Harbottle ${ }^{b}$ and Jae W. Lee (DD *a
Potassium copper hexacyanoferrate (KCUHCF)-incorporated magnetic chitosan beads (HMC) were synthesized for both selective $\mathrm{Cs}^{+}$removal in aqueous solutions and facile recovery of the spent adsorbent. To disperse and immobilize large amounts of the KCuHCF, methyl acrylate and diethylenetriamine were sequentially grafted onto the one-step synthesized magnetic chitosan beads. The additional introduction of amino functionality led to the enriched $\mathrm{Cu}^{2+}$ ions on the bead surface to incorporate KCUHCF into the grafting matrix. Consequently, the HMC exhibited a high $\mathrm{Cs}^{+}$capacity calculated to be $136.47 \mathrm{mg} \mathrm{g}^{-1}$ from the Langmuir model, and the equilibrium was established within $4 \mathrm{~h}$. Moreover, the HMC exhibited excellent stability in a wide $\mathrm{pH}$ range from 4 to 11 and an outstanding $\mathrm{Cs}^{+}$selectivity $(>97 \%)$ in seawater $\left(1.11 \mathrm{mg} \mathrm{L}^{-1} \mathrm{Cs}^{+}\right)$. From a practical point of view, the HMC was stable during five successive adsorption cycles and easily recovered by magnets, enabling continuous operation to decontaminate a large volume of wastewater.
\end{abstract}

\section{Introduction}

As the demands for more efficient and economical energy increase, nuclear power has become a promising candidate instead of thermal power derived from fossil fuels due to low $\mathrm{CO}_{2}$ emissions. ${ }^{1}$ Although nuclear power has become an important energy source providing approximately $11 \%$ of the worldwide electricity under stringent government regulations, it is considered a threat that needs substantial environmental regulations, especially after accidents such as the Fukushima Daiichi nuclear power plant accident. ${ }^{2}$ This disaster caused the release and dispersion of a significant amount of radioactive nuclides into different environments such as the soil and water. ${ }^{3,4}$ Among the radionuclides, ${ }^{137} \mathrm{Cs}$ was the most problematic fission product because of its long half-life around 30 years, high-energy gamma-ray emissions, and high solubility in water. ${ }^{5}$ Moreover, its chemical similarity with potassium ions causes $\mathrm{Cs}^{+}$ions to spread into the ecosystem; hence, the radioactive $\mathrm{Cs}^{+}$ions should be decontaminated safely and effectively from the environment. ${ }^{6}$

A variety of purification methods such as co-precipitation, solvent extraction, adsorption, and chromatography have been

${ }^{a}$ Department of Chemical and Biomolecular Engineering, Korea Advanced Institute of Science and Technology (KAIST), Daejeon 305-701, Republic of Korea. E-mail: jaewlee@kaist.ac.kr; Tel: +82-42-350-3940

${ }^{b}$ School of Chemical and Process Engineering, University of Leeds, Leeds LS2 9JT, UK

$\dagger$ Electronic supplementary information (ESI) available: Detailed description for experimental and characterization (EDS and XPS). See DOI: 10.1039/c8ra09386e investigated to separate $\mathrm{Cs}^{+}$ions from the contaminated water. However, traditional methods such as thermal treatment or solvent extraction are limited due to the operational cost and the inability of a large-area remediation of water in the nuclear industry. ${ }^{7}$ Therefore, adsorption is one of the most efficient methods for large-scale applications of wastewater treatment, and especially for $\mathrm{Cs}^{+}$adsorption, a number of organic and inorganic materials such as carbon, ${ }^{3,8}$ zeolite, ${ }^{9}$ clays, ${ }^{10,11}$ and layered metal thiophosphate ${ }^{12}$ have been used. Nevertheless, the presence of large amounts of competing cations such as $\mathrm{Na}^{+}, \mathrm{K}^{+}, \mathrm{Ca}^{2+}$, and $\mathrm{Mg}^{2+}$ still makes it a challenge to separate diluted $\mathrm{Cs}^{+}$ions from wastewater using these common inorganic adsorbents.

Meanwhile, transition metal hexacyanoferrates (MHCFs), which can simply be synthesized by a precipitation method, have been widely reported for efficient $\mathrm{Cs}^{+}$removal with their high selectivity even in competitive aqueous environments.,13 The high affinity of the MHCFs for $\mathrm{Cs}^{+}$ion originates from their lattice spacing which is similar to the size of hydrated $\mathrm{Cs}^{+}$ion $(3.25 \AA) .{ }^{14,15}$ However, the MHCFs are a very fine powder that makes it difficult to recover the adsorbent particles from water by either centrifugation or filtration; thus, immobilizing the MHCFs in supporting materials is required for practical applications. For this approach, various polymers such as carboxymethyl cellulose, ${ }^{16,17}$ polyvinyl alcohol, ${ }^{18-21}$ alginate, ${ }^{22}$ and chitosan, ${ }^{23,24}$ have been studied as supporting materials. These polymeric materials have abundant functional groups on their surface which are highly effective for both incorporating the 
MHCF nanoparticles and further processing for commercial use.

Among these polymer matrices, chitosan-based adsorbents have strong advantages of cost-effectiveness, resource abundance, and many functional groups for desalination by ion adsorption. ${ }^{25-27}$ Particularly, numerous amino functional groups in chitosan contribute to the coordination of various metal ions. ${ }^{28,29}$ This unique property enables copper ions to be well dispersed, and thus, potassium copper hexacyanoferrate (KCuHCF) can be immobilized by precipitation on the surface of chitosan-based adsorbents for effective $\mathrm{Cs}^{+}$removal. However, pure chitosan has a poor mechanical strength and low acidic stability, making it difficult to use in a real adsorption process. $^{30-33}$ Previous studies have mainly focused on the crosslinking of chitosan to enhance the stability of the material, ${ }^{34}$ but this approach has a disadvantage of a reduced amino functionality due to the crosslinking reaction, eventually limiting the introduction of active sites and the adsorption performance of the chitosan.

In this work, KCuHCF-incorporated magnetic chitosan beads functionalized with amine were fabricated to improve the $\mathrm{Cs}^{+}$removal performance by the introduction of additionally available ion binding sites. Instead of crosslinking, the magnetic $\mathrm{Fe}_{3} \mathrm{O}_{4}$ nanoparticle-containing chitosan beads were synthesized by a one-step co-precipitation method to improve the chemical stability. ${ }^{35,36}$ This strategy has an advantage in that the used adsorbent can be efficiently recovered by simple magnetic separation after the water purification process. ${ }^{37,38}$ Additionally, simple grafting of methyl acrylate and diethylenetriamine was done by the Michael reaction and amidation, ${ }^{39-42}$ in sequence, to increase the amino functionality in the chitosan matrix. The high content of amino groups in the chitosan beads contributed to the enhancement of the $\mathrm{Cu}^{2+}$ coordination, and this is advantageous for the subsequent immobilization of large amounts of KCuHCF, which is synthesized by simple co-precipitation. As a result, well-dispersed KCuHCF and $\mathrm{Fe}_{3} \mathrm{O}_{4}$ nanoparticles were successfully coimmobilized in the final chitosan bead structure to achieve both a high performance and facile recovery, respectively. ${ }^{43}$ The $\mathrm{Cs}^{+}$removal efficiency of the adsorbent was evaluated in terms of the adsorption capacity, selectivity, kinetics, pH stability, and reusability. Moreover, various physicochemical characterizations were conducted to determine the effect of both the magnetic property and amino-functionalization on the excellent $\mathrm{Cs}^{+}$separation performance of the adsorbent.

\section{Experimental}

Chemicals, fabrication of the magnetic nanoparticle-containing chitosan beads (MC), characterization, and $\mathrm{Cs}^{+}$adsorption test depending on the adsorbent dose, $\mathrm{pH}$, and reusability test are provided in the ESI. $\dagger$

\subsection{Synthesis of the amino-functionalized MC (AMC)}

The amino-functionalized MC (AMC) was synthesized by modifying the procedures reported in a previous study (Fig. 1). ${ }^{44}$
To introduce more amino groups on the surface of the adsorbent, methyl acrylate was used to connect between the MC and DETA. Methyl acrylate was grafted to the amino groups on the surface of the MC by the Michael addition reaction..$^{41}$ Briefly, $1 \mathrm{~g}$ of the MC and $120 \mathrm{~mL}$ of methanol were mixed into a 2-neckround flask. Then, the mixture was continuously stirred and heated to $50{ }^{\circ} \mathrm{C}$ under reflux with purging nitrogen gas, and $6 \mathrm{~mL}$ of methyl acrylate was added to the flask using a syringe. After $24 \mathrm{~h}$, the methyl acrylate-grafted MC beads were washed with methanol several times and dried at $35^{\circ} \mathrm{C}$. Then, DETA was grafted to the terminal ester groups on the surface of the methyl acrylate-grafted MC by the amidation reaction. ${ }^{39}$ All the methyl acrylate-grafted $\mathrm{MC}$ and $150 \mathrm{~mL}$ of methanol were added into a 2-neck-round flask, and the mixture was agitated at $50{ }^{\circ} \mathrm{C}$ under reflux in a nitrogen atmosphere. After stabilizing this reaction condition for $1 \mathrm{~h}, 20 \mathrm{~mL}$ of DETA was added to the flask, and the reaction was carried out for $36 \mathrm{~h}$. Finally, the prepared AMC was washed with methanol and subsequently freeze-dried.

\subsection{Synthesis of the KCuHCF-immobilized AMC (HMC)}

The as-synthesized AMC was immersed in $0.16 \mathrm{~mol} \mathrm{~L}^{-1}$ of copper(II) sulfate solution under stirring for $10 \mathrm{~h}$ to immobilize the $\mathrm{Cu}^{2+}$ ions to the amino groups on the AMC surface. The $\mathrm{Cu}-$ incorporated AMC was separated by a magnet and washed with DI water several times. Then, the $\mathrm{Cu}$-incorporated AMC was put into $0.16 \mathrm{~mol} \mathrm{~L}^{-1}$ of a prepared potassium hexacyanoferrate solution under stirring to conduct KCuHCF formation via coprecipitation. After $10 \mathrm{~h}$ of contact, the KCuHCF-immobilized AMC (HMC) was collected by a magnet and washed with DI water several times and finally freeze-dried.

\section{3 $\mathrm{Cs}^{+}$adsorption experiment}

All the cesium (inactive cesium, ${ }^{133} \mathrm{Cs}$ ) adsorption studies were performed using a batch-shaking method. Thus, $20 \mathrm{mg}$ of the HMC was put into the $\mathrm{Cs}^{+}$solution which had a specific concentration. For the $\mathrm{Cs}^{+}$ion-exchange experiments, the mixture was shaken for $24 \mathrm{~h}$ at room temperature. The initial and specific concentrations of the $\mathrm{Cs}^{+}$ions in the aqueous phase were precisely measured using an inductively coupled plasma mass spectrometer (ICP-MS, Agilent ICP-MS 7700S).

To determine the adsorption isotherm, the HMC was shaken in a $\mathrm{Cs}^{+}$solution by varying the initial $\mathrm{Cs}^{+}$concentration $\left(C_{0}\right)$ from 5 to $500 \mathrm{mg} \mathrm{L}^{-1}\left(m / V=1 \mathrm{~g} \mathrm{~L}^{-1}, m(\mathrm{~g})\right.$ is the mass of the dried adsorbent, and $V(\mathrm{~L})$ is the volume of the $\mathrm{Cs}^{+}$solution.). After $24 \mathrm{~h}$, the residual $\mathrm{Cs}^{+}$concentration $\left(C_{\mathrm{e}}\right)$ in the filtrate was quantified with an ICP-MS.

The adsorption kinetics of cesium from DI water was investigated by immersing $200 \mathrm{mg}$ of the HMC in $8.99 \mathrm{mg} \mathrm{L}^{-1}$ of the $\mathrm{Cs}^{+}$solution $\left(m / V=1 \mathrm{~g} \mathrm{~L}^{-1}\right)$ at room temperature. The shaking sample was stopped at a specific time from $1 \mathrm{~min}$ up to $24 \mathrm{~h}$, and $1 \mathrm{~mL}$ of the solution was extracted, and the $\mathrm{Cs}^{+}$concentration was determined.

The $\mathrm{Cs}^{+}$adsorption amount $\left(Q_{\mathrm{e}}, \mathrm{mg} \mathrm{g}^{-1}\right)$, the removal efficiency $(\mathrm{RE}, \%)$, and the distribution coefficient $\left(K_{\mathrm{d}}, \mathrm{mL} \mathrm{g}^{-1}\right)$ were calculated using the following equations: 

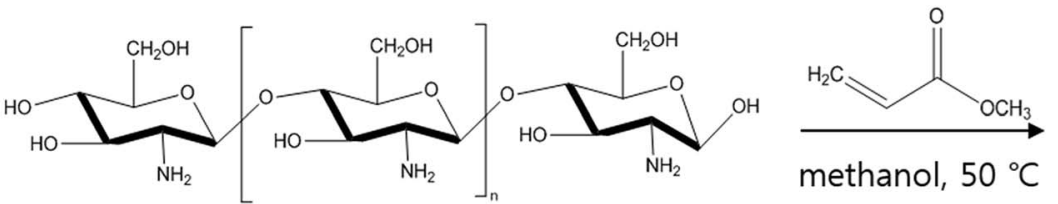

\section{chitosan backbone in the MC}
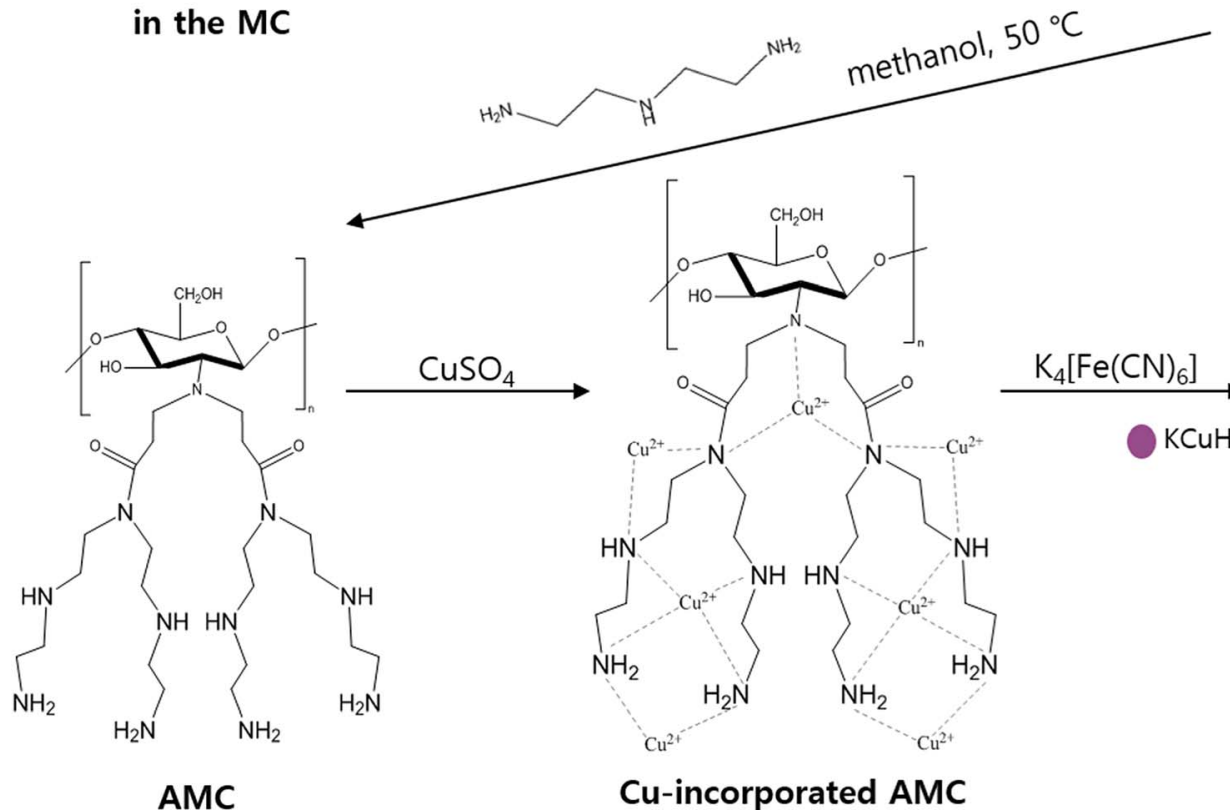

\section{methyl acrylate- grafted MC}

Fig. 1 Schematic representation for the HMC synthesis.

$$
\begin{aligned}
Q_{\mathrm{e}} & =\left(C_{0}-C_{\mathrm{e}}\right)\left(\frac{V}{m}\right) \\
\mathrm{RE} & =\frac{C_{0}-C_{t}}{C_{0}} \times 100 \% \\
K_{\mathrm{d}} & =\frac{C_{0}-C_{\mathrm{e}}}{C_{\mathrm{e}}}\left(\frac{V}{m}\right)
\end{aligned}
$$

where $C_{0}$ and $C_{\mathrm{e}}$ are the initial and equilibrium concentrations of the $\mathrm{Cs}^{+}\left(\mathrm{mg} \mathrm{L}^{-1}\right)$, respectively, and $C_{t}$ indicates the concentration $\left(\mathrm{mg} \mathrm{L}^{-1}\right)$ after the designated time $(t)$.

\section{Results and discussion}

\subsection{Characterization: textural properties}

The morphologies of the beads are shown in the SEM images (Fig. 2). Fig. 2(a) and (b) show the network structure of the chitosan polymer and the rough surface of the MC. After grafting the methyl acrylate and DETA onto the surface of the beads, the KCuHCF nanoparticles were successfully immobilized onto the surface of the AMC evident by the SEM images of the HMC (Fig. 2(c) and (d)). EDS analysis was conducted to investigate the composition of the MC and HMC (Fig. S1 $\dagger$ ). The EDS spectra of the MC showed peaks for $\mathrm{C}, \mathrm{O}, \mathrm{N}$, and $\mathrm{Fe}$, which are the four main components of the chitosan and $\mathrm{Fe}_{3} \mathrm{O}_{4}$. On the other hand, the EDS spectra of the HMC showed peaks for $\mathrm{C}$,
$\mathrm{O}, \mathrm{N}, \mathrm{Fe}, \mathrm{Cu}$, and $\mathrm{K}$, indicating that the KCuHCF was incorporated into the beads.

The thermal stability of the MC and HMC was investigated by thermogravimetric analysis (TGA) under a nitrogen atmosphere (Fig. 3(a)). There were three stages of weight loss in the TGA curve for the MC, and the first stage with about $2.5 \%$ weight loss to $200{ }^{\circ} \mathrm{C}$ was due to the elimination of adsorbed water. The other two major weight losses occurred at $250-400{ }^{\circ} \mathrm{C}$ and $600-750{ }^{\circ} \mathrm{C}$, which indicate the degradation of the

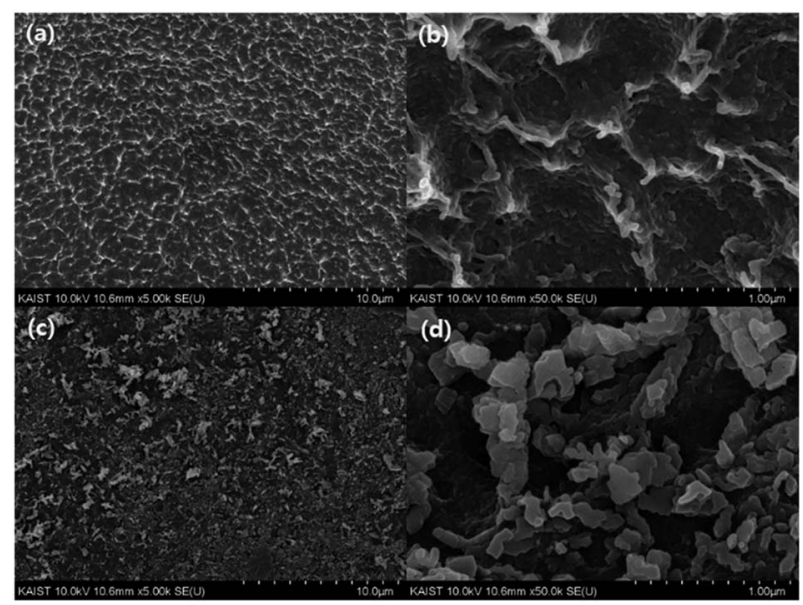

Fig. 2 SEM images of (a) and (b) the MC, and (c) and (d) the HMC. 

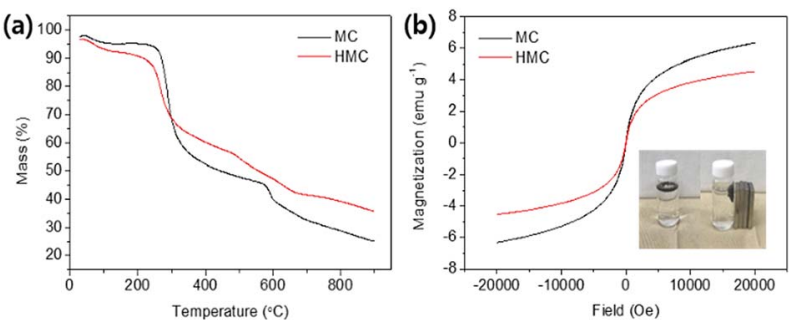

Fig. 3 (a) TGA profiles of the MC and HMC. (b) Magnetization curves of the $M C$ and $\mathrm{HMC}$ depends on field $(\mathrm{Oe})$, and images of the $\mathrm{HMC}$ before and after separation by magnets (inset).

backbone chains of the chitosan and further slow decomposition of the residual chitosan or the intermediates from the predegraded chitosan. ${ }^{45,46}$ After the decomposition of the organic phase in the MC over $800{ }^{\circ} \mathrm{C}$, it is certain that the residual components are due to the incorporated $\mathrm{Fe}_{3} \mathrm{O}_{4}$. As shown in Fig. 3(a), the TGA profile of the HMC was similar to the curve for the MC, but there was a shift of the second step around $200{ }^{\circ} \mathrm{C}$ in the degradation and another weight loss at $300-550{ }^{\circ} \mathrm{C}$. The shift is due to the presence of the $\mathrm{KCuHCF},{ }^{47}$ and the weight loss corresponds to the decomposition of the cyano groups in the KCuHCF. ${ }^{16}$ Furthermore, the mass fraction of the organic components in the HMC was approximately estimated to be $60.75 \%$, and the rest is related to the inorganic elements from the $\mathrm{Fe}_{3} \mathrm{O}_{4}$ and $\mathrm{KCuHCF}$ in the beads.

The content of the $\mathrm{Fe}_{3} \mathrm{O}_{4}$ and KCuHCF in the MC and HMC was analyzed by measuring the weight percentages of $\mathrm{Fe}, \mathrm{K}$ and $\mathrm{Cu}$ using ICP-OES. Table 1 shows the calculated amounts of the $\mathrm{Fe}_{3} \mathrm{O}_{4}$ and KCuHCF in the beads. Furthermore, based on the charge neutrality and stoichiometric ratio of the elements, the estimated chemical composition of the KCuHCF in the HMC structure was $\mathrm{K}_{0.57} \mathrm{Cu}_{1.71}\left[\mathrm{Fe}(\mathrm{CN})_{6}\right]$.

The magnetic properties of the MC and HMC were investigated using the magnetization curves acquired from the vibrating-sample magnetometer (VSM) analysis. As shown in Fig. 3(b), the magnetization curves for both the MC and HMC beads represented superparamagnetic characteristics with zero coercivity and zero remanence. ${ }^{46}$ The magnetization values of the MC and HMC were 6.32 and $4.54 \mathrm{emu} \mathrm{g}^{-1}$ at $20000 \mathrm{Oe}$, respectively. The lower mass normalized magnetization value of the HMC rather than that of the MC is associated with the incorporation of KCuHCF. As shown in the inset of Fig. 3(b), however, the HMC was attracted to the wall of the vial immediately by placement of the magnets, indicating that the HMC could be easily separated from the aqueous solution after the $\mathrm{Cs}^{+}$adsorption.

Table 1 ICP-OES elemental analysis (wt\%)

\begin{tabular}{llllll}
\hline & $\mathrm{Cu}$ & $\mathrm{Fe}$ & $\mathrm{K}$ & $\mathrm{Fe}_{3} \mathrm{O}_{4}$ & KCuHCF \\
\hline $\mathrm{MC}$ & - & 14.222 & - & 19.646 & - \\
$\mathrm{HMC}$ & 6.3045 & 11.801 & 1.298 & 11.827 & 19.881
\end{tabular}

\subsection{Characterization: chemical properties}

The co-immobilization of crystalline $\mathrm{Fe}_{3} \mathrm{O}_{4}$ and KCuHCF was confirmed by X-ray diffraction (XRD) (Fig. 4(a)). The characteristic peaks at $30.5^{\circ}, 35.5^{\circ}, 43.4^{\circ}, 57.2^{\circ}$, and $62.7^{\circ}$ indicate the presence of $\mathrm{Fe}_{3} \mathrm{O}_{4}$ nanoparticles in the MC. ${ }^{48}$ These peaks show that the co-precipitation of chitosan and $\mathrm{Fe}_{3} \mathrm{O}_{4}$ for the fabrication of the magnetic beads did not change the phase of the $\mathrm{Fe}_{3} \mathrm{O}_{4}$ nanoparticles. Compared to the MC, the HMC has eight distinguishable peaks at $2 \theta=17.7^{\circ}, 25^{\circ}, 36^{\circ}, 40^{\circ}, 44^{\circ}, 52^{\circ}, 55^{\circ}$, and $58^{\circ}$, and these peaks are well matched with the KCuHCF pattern (PDF no. 01-075-0023). Although some major peaks of the $\mathrm{Fe}_{3} \mathrm{O}_{4}$ nanoparticles are overlapped with those of the $\mathrm{KCuHCF}$, the characteristic peak at $30^{\circ}$ supports the presence of $\mathrm{Fe}_{3} \mathrm{O}_{4}$, along with the magnetic properties revealed by the VSM result. Thus, the XRD patterns show the successful incorporation of the $\mathrm{Fe}_{3} \mathrm{O}_{4}$ nanoparticles and KCuHCF into the chitosan bead structure.

Moreover, the average crystal size of the $\mathrm{Fe}_{3} \mathrm{O}_{4}$ and KCuHCF in the beads was determined by the Scherrer equation:

$$
L=\frac{K \lambda}{\beta \cos \theta}
$$

where $K$ is a dimensionless factor for the crystal shape (varying between 0.9 and 1 , here taken as 0.94 ); $\lambda$ is the X-ray wavelength $(\lambda=1.54 \AA$ for $\mathrm{Cu} \mathrm{K} \alpha) ; \theta$ is the Bragg diffraction angle at the specific peak (rad), and $\beta$ is the line broadening at half of the maximum intensity (FWHM, full width at half maximum, rad). The calculated average size of $\mathrm{Fe}_{3} \mathrm{O}_{4}$ from the major peaks at $30.5^{\circ}, 35.5^{\circ}$, and $43.4^{\circ}$ was approximately $4-4.6 \mathrm{~nm}$, and KCuHCF from the major peaks at $17.7^{\circ}, 25^{\circ}$, and $36^{\circ}$ was approximately $25 \mathrm{~nm}$.

The FT-IR spectra of the chitosan powder, MC, methyl acrylate-grafted MC, AMC, and HMC were obtained to verify the successful reactions in each step (Fig. 4(b)). For the chitosan powder, the main peaks at $895 \mathrm{~cm}^{-1}$ and $1155 \mathrm{~cm}^{-1}$ are attributed to the saccharide, and the peak at $1654 \mathrm{~cm}^{-1}$ is assigned to the $-\mathrm{NH}$ absorption peak of the amine group $\left(-\mathrm{NH}_{2}\right)$ in the chitosan polymer backbone. The spectrum obtained from the MC revealed a characteristic band at $560 \mathrm{~cm}^{-1}$, corresponding to the $\mathrm{Fe}-\mathrm{O}$ stretching vibrational mode of $\mathrm{Fe}_{3} \mathrm{O}_{4} \cdot{ }^{49}$ The MC showed a new absorption peak at $1719 \mathrm{~cm}^{-1}$ which was related to the formation of the ester group $(-\mathrm{O}-\mathrm{C}=\mathrm{O}-)$ resulting from the grafting of methyl acrylate by the Michael reaction. In addition, after grafting DETA to the beads, the newly generated
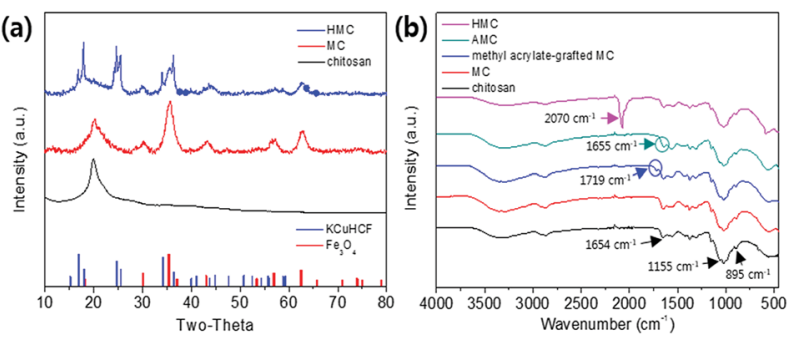

Fig. 4 (a) XRD patterns of the MC and HMC and (b) FT-IR spectra of beads in each synthesis step. 
peak at $1655 \mathrm{~cm}^{-1}$ is related to the formation of the amide bond (-CONH-), and simultaneously, the disappearance of peak at $1719 \mathrm{~cm}^{-1}$ showed the successful reaction between the ester group of the methyl acrylate-grafted $\mathrm{MC}$ and the amino group of the DETA. ${ }^{44}$ The presence of a strong peak at $2070 \mathrm{~cm}^{-1}$ for the $\mathrm{HMC}$ is due to $\mathrm{C} \equiv \mathrm{N}$ stretching vibration from the immobilized KCuHCF. Importantly, the HMC also included the peaks for the chitosan and $\mathrm{Fe}_{3} \mathrm{O}_{4}$, revealing the successful introduction of the amino functionality and KCuHCF in the magnetic chitosan structure.

XPS analyses were conducted to identify the active sites for the formation of KCuHCF because the HMC has immense functional groups including $\mathrm{N}$ and $\mathrm{O}$ atoms such as amine, hydroxyl, and amide, which $\mathrm{Cu}^{2+}$ ions can be coordinated to. The XPS survey results show that the HMC contained $\mathrm{C}, \mathrm{O}, \mathrm{N}$, $\mathrm{Cu}, \mathrm{Fe}$, and $\mathrm{K}$ (refer to Fig. S2(a)†). The N 1s peaks at 399.34 and $400.11 \mathrm{eV}$ (Fig. 5(a)) are assigned to free amine groups $\left(\mathrm{NH}_{2}\right)$ and $\mathrm{NH}_{2}-\mathrm{O}$ in the $\mathrm{MC}$, respectively. ${ }^{50}$ Compared to the peaks of Fig. 5(a), the N 1s spectrum of the HMC in Fig. 5(b) has peaks at $397.7,399.62$ and $401.89 \mathrm{eV}$ which correspond to the $\mathrm{C} \equiv \mathrm{N}$ groups in the $\mathrm{KCuHCF},-\mathrm{N}-$, and protonated amine groups because of the chelation with $\mathrm{Cu}^{2+}$ ions after incorporation of the KCuHCF, respectively. ${ }^{51,52}$ However, the $\mathrm{O}$ 1s spectra (Fig. S2(b) and (c) $\dagger$ ) for both the MC and HMC did not have any changes after the KCuHCF immobilization. In other words, $\mathrm{Cu}^{2+}$ ions, which are the precursors of the KCuHCF, chelated with the $\mathrm{N}$ atoms of the bead surface; thus, the introduction of amino groups using methyl acrylate and DETA onto the beads has a crucial role in the immobilization of KCuHCF.

\section{3 $\mathrm{Cs}^{+}$adsorption studies}

3.3.1 Effect of adsorbent dose. Fig. 6(a) shows the removal efficiency and adsorbed amount of $\mathrm{Cs}^{+}\left(C_{0}=10 \mathrm{mg} \mathrm{L}^{-1}\right)$ at different adsorbent doses. It is shown that as the dose increased, the removal efficiency increased from $69 \%$ to $99 \%$, and all of the results above a dosage of $1 \mathrm{~g} \mathrm{~L}^{-1}$ show a similarly high removal over $98 \%$. On the other hand, the adsorption capacity decreased as the dosage increased, indicating unsaturated adsorption sites in the HMC in the low $\mathrm{Cs}^{+}$concentration. Therefore, considering both the removal efficiency and adsorbed amount results, the remaining adsorption experiments were conducted with $1 \mathrm{~g} \mathrm{~L}^{-1}$ unless otherwise stated.

3.3.2 Effect of $\mathbf{p H}$. In general, the $\mathrm{pH}$ of the aqueous solution is one of the most important factors affecting the adsorption performance by changing the surface properties of
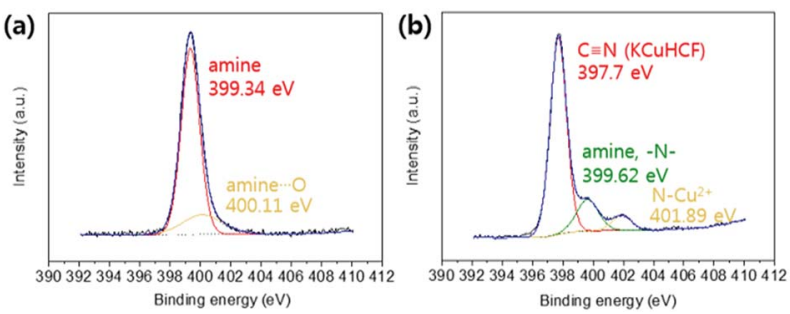

Fig. 5 XPS N 1s spectra of the (a) MC and (b) HMC.
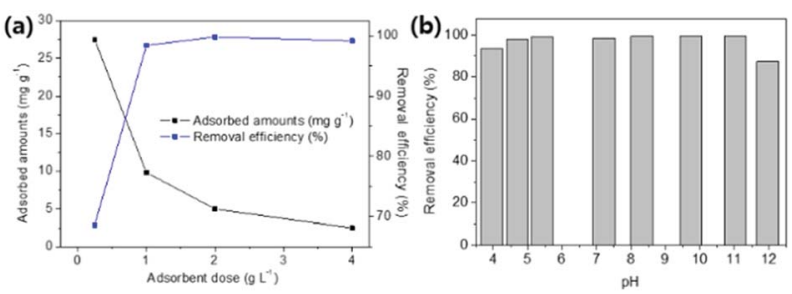

Fig. 6 (a) Effect of adsorbent dose on removal efficiency (\%) and adsorbed amounts $\left(\mathrm{mg} \mathrm{g}^{-1}\right.$ ). (b) Effect of $\mathrm{pH}$ of solution on $\mathrm{Cs}^{+}$ adsorption in DI water.

the beads. Fig. 6(b) indicates the $\mathrm{Cs}^{+}$removal efficiency was not significantly influenced by the $\mathrm{pH}$ values. The initial $\mathrm{Cs}^{+}$ concentration was $9.69 \mathrm{mg} \mathrm{L}^{-1}$, and the $\mathrm{pH}$ range was from 4 to 12. In acidic conditions from $\mathrm{pH} 4$ to 5.5 , the removal efficiency was higher than $94 \%$. It demonstrated that amino-modification by grafting methyl acrylate and DETA improved acidic stability of chitosan through the enhanced intra- and intermolecular hydrogen bonds. ${ }^{44}$ In basic conditions at a $\mathrm{pH}$ between 8.3 and 11, the HMC showed over 99\% removal; however, at $\mathrm{pH} 11.96$, the removal efficiency decreased to $87 \%$. This could be explained by the fact that the metal hexacyanoferrate tends to decompose under high alkaline conditions as previously reported. ${ }^{52,53}$ These results show that the HMC is able to be used in the remediation of contaminated water with a broad $\mathrm{pH}$ range.

3.3.3 Adsorption isotherm. The $\mathrm{Cs}^{+}$adsorption isotherm was obtained to evaluate the removal capacity and the affinity of the HMC using different initial concentrations of $\mathrm{Cs}^{+}$solution. To evaluate the thermodynamic parameters, the experimental data were fitted with well-known Langmuir and Freundlich isotherm models (Fig. 7(a)). The equations are described as follow:

Langmuir isotherm

$$
Q_{\mathrm{e}}=\frac{Q_{\mathrm{m}} b C_{\mathrm{e}}}{1+b C_{\mathrm{e}}}
$$

Freundlich isotherm

$$
Q_{\mathrm{e}}=K_{\mathrm{F}} C_{\mathrm{e}}^{1 / n}
$$

where $Q_{\mathrm{e}}\left(\mathrm{mg} \mathrm{g}^{-1}\right)$ and $Q_{\mathrm{m}}\left(\mathrm{mg} \mathrm{g}^{-1}\right)$ represent the adsorption capacity of the $\mathrm{Cs}^{+}$at the equilibrium and the maximum
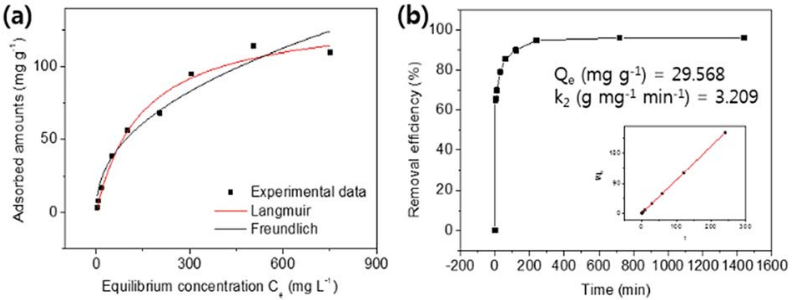

Fig. 7 (a) The $\mathrm{Cs}^{+}$adsorption isotherm curve and (b) adsorption kinetics and plot of $t / Q_{t} v s$. $t$ for the HMC. 
adsorption capacity, respectively. $C_{\mathrm{e}}\left(\mathrm{mg} \mathrm{\textrm {L } ^ { - 1 }}\right)$ is the $\mathrm{Cs}^{+}$ concentration at the equilibrium in the liquid phase; $b\left(\mathrm{~L} \mathrm{mg}^{-1}\right)$ is an affinity coefficient, and $K_{\mathrm{F}}$ and $1 / n$ are the Freundlich parameters related to the adsorption capacity and intensity.

As presented in Table 2, the isotherm parameters were calculated by the non-linear curve fitting of the above equations. The adsorption process of the HMC agreed better with the Langmuir model with a higher correlation coefficient of 0.98 , and the Freundlich heterogeneity parameter is around 2.27 ( $n>$ 1). These results suggest the uniform distribution of the KCuHCF nanoparticles in the HMC and the chemical binding of $\mathrm{Cs}^{+}$by the active sites. Based on the Langmuir isotherm model, the HMC provided a superior maximum adsorption capacity of $136.47 \mathrm{mg} \mathrm{g}^{-1}$ compared to those of the other $\mathrm{Cs}^{+}$adsorbents containing magnetic nanoparticles (Table 3 ). It can be attributed to the increase of amino functionality, which resulted in the incorporation of a comparatively great quantity of KCuHCF particles, leading to the superior $\mathrm{Cs}^{+}$adsorption performance.

3.3.4 Adsorption kinetics. The adsorption kinetics was studied to determine the removal efficiency. Fig. 7(b) shows the removal rate of $\mathrm{Cs}^{+}$using the same amount of the $\mathrm{HMC}\left(\mathrm{Cs}^{+}\right.$ initial concentration $=8.99 \mathrm{mg} \mathrm{L}^{-1}$ ) over a specific contact time. The HMC exhibited a fast uptake process to remove $90 \%$ of the $\mathrm{Cs}^{+}$ions within $2 \mathrm{~h}$ and greater than $95 \%$ of the $\mathrm{Cs}^{+}$within $4 \mathrm{~h}$. The time-dependent $\mathrm{Cs}^{+}$removal data were fitted with a linear pseudo-second-order kinetic model to obtain the specific parameters regarding the adsorption kinetics. Pseudo-secondorder kinetics equation is as follows:

$$
\frac{t}{Q_{t}}=\frac{1}{k_{2} Q_{\mathrm{e}}}+\frac{t}{Q_{\mathrm{e}}}
$$

where $Q_{\mathrm{e}}\left(\mathrm{mg} \mathrm{g}^{-1}\right)$ and $Q_{t}\left(\mathrm{mg} \mathrm{g}^{-1}\right)$ are the equilibrium adsorption capacity and the adsorbed amount at a given time $t$ (min), respectively, and $k_{2}\left(\mathrm{~g} \mathrm{mg}^{-1} \mathrm{~min}^{-1}\right)$ is adsorption rate constant of the pseudo-second-order kinetic model. The parameters $k_{2}$ and $Q_{\mathrm{e}}$ were calculated (Fig. 7(b)) from the slope and intercept of $t / Q_{t}$ versus $t$. The fitting results indicate that the rate determining step of the metal ion was mainly governed by the chemical adsorption onto the adsorbent. ${ }^{57,58}$

3.3.5 Competitive adsorption. To investigate the performance in more realistic conditions, the $\mathrm{Cs}^{+}$removal test was also done using seawater $\left(\mathrm{Na}^{+}: 8,247, \mathrm{~K}^{+}: 286, \mathrm{Ca}^{2+}: 179\right.$, and $\mathrm{Mg}^{2+}: 1246 \mathrm{mg} \mathrm{L}^{-1}$ refer to Table S1 $\dagger$ ). The removal efficiency and distribution coefficient of the HMC are summarized in Table 4. The HMC has not only extraordinary removal efficiency in pure water, but also excellent $\mathrm{Cs}^{+}$selectivity even in seawater which includes an extremely high concentration of competitive ions. The distribution coefficient represents the $\mathrm{Cs}^{+}$binding

Table 2 Langmuir and Freundlich isotherm parameters

\begin{tabular}{|c|c|c|c|c|c|}
\hline \multicolumn{3}{|c|}{ Langmuir isotherm parameters } & \multicolumn{3}{|c|}{$\begin{array}{l}\text { Freundlich isotherm } \\
\text { parameters }\end{array}$} \\
\hline$Q_{\mathrm{m}}\left(\mathrm{mg} \mathrm{g}^{-1}\right)$ & $b\left(\mathrm{~L} \mathrm{mg}^{-1}\right)$ & $R^{2}$ & $n$ & $K_{\mathrm{F}}\left(\mathrm{L} \mathrm{g}^{-1}\right)$ & $R^{2}$ \\
\hline 136.47 & 0.007 & 0.98 & 2.27 & 6.69 & 0.9 \\
\hline
\end{tabular}

Table 3 Comparison of $\mathrm{Cs}^{+}$adsorption capacity with different magnetic adsorbents

\begin{tabular}{lcl}
\hline Adsorbents & $\begin{array}{l}\text { Adsorption capacity } \\
\left(\mathrm{mg} \mathrm{g}^{-1}\right)\end{array}$ & Ref. \\
\hline $\mathrm{NaCuHCF}$ & 125 & 54 \\
$\mathrm{PTH}$ & 68.71 & 55 \\
$\mathrm{MHPVA}$ & 82.8 & 18 \\
$\mathrm{~PB}_{\mathrm{Fe}} \mathrm{O}_{4} / \mathrm{GO}$ & 55.56 & 53 \\
Magnetic chitosan & 3.86 & 56 \\
PB-coated MNP & 96 & 57 \\
HMC & 136.47 & This work
\end{tabular}

affinity to the adsorbent, and a higher value indicates a stronger ion exchange. A $K_{\mathrm{d}}$ value on the order of $10^{4}$ to $10^{5} \mathrm{~mL} \mathrm{~g}^{-1}$ suggests an exceptionally strong affinity, ${ }^{59}$ and these values for the HMC in seawater were $1.75 \times 10^{4} \mathrm{~mL} \mathrm{~g}^{-1}\left(\mathrm{Cs}^{+} 10.56 \mathrm{mg} \mathrm{L}^{-1}\right)$ and $3.27 \times 10^{4} \mathrm{~mL} \mathrm{~g}^{-1}\left(\mathrm{Cs}^{+} 1.11 \mathrm{mg} \mathrm{L}^{-1}\right)$.

The $K_{\mathrm{d}}$ values of $\mathrm{Cs}^{+}$on the HMC in seawater were as good as those in the DI water, and the other excess cations (e.g. $, \mathrm{Na}^{+}, \mathrm{K}^{+}$, $\mathrm{Ca}^{2+}$, and $\mathrm{Mg}^{2+}$ ) did not highly affect the binding of $\mathrm{Cs}^{+}$. In terms of the removal efficiency, more than $94 \%$ of the $\mathrm{Cs}^{+}$was removed even in the seawater condition, indicating that the HMC is a highly efficient candidate to remediate nuclearcontaminated water in which accidentally released $\mathrm{Cs}^{+}$ions are diluted. These promising results show that the welldispersed KCuHCF nanoparticles on the amino groups in the HMC could contribute to the remarkable $\mathrm{Cs}^{+}$selectivity even in highly competitive conditions.

3.3.6 Reusability of the adsorbent. The adsorption performance depending on the reusability is crucial for commercial applications. After the adsorption of $\mathrm{Cs}^{+}$, the recovered HMC by a magnet was successively reused for the next adsorption test in the absence of HMC regeneration (Fig. 8(a)). The $\mathrm{Cs}^{+}$adsorption test was repeated five times under the same condition $(\mathrm{m} / \mathrm{V}=4 \mathrm{~g}$ $\mathrm{L}^{-1}, 12 \mathrm{~h}$ contact time, and $0.86 \mathrm{mg} \mathrm{L}^{-1} \mathrm{Cs}^{+}$in seawater). As shown in Fig. 8(b), the removal efficiency of $\mathrm{Cs}^{+}$reached $99.5 \%$ in the first adsorption and was maintained above $90 \%$ until the fourth cycle without the regeneration of the HMC. After the fifth adsorption cycle, the $\mathrm{Cs}^{+}$removal efficiency slightly decreased to about $90 \%(88.4 \%)$, but the HMC was sustainable to be separated by magnets. This result shows that the HMC is remarkably durable after repeated use and can selectively remove trace $\mathrm{Cs}^{+}$ions in seawater; hence, the HMC can purify a large volume of contaminated water in a continuous process, resulting in a significant waste volume reduction. Furthermore,

Table 4 Removal efficiency and $K_{d}$ value for the HMC in DI water and seawater

\begin{tabular}{lcll}
\hline & & $\begin{array}{l}\text { Removal efficiency } \\
(\%)\end{array}$ & $K_{\mathrm{d}}\left(\mathrm{mL} \mathrm{g}^{-1}\right)$ \\
\hline \multirow{2}{*}{ DI water } & 8.01 & 99.1 & $1.13 \times 10^{5}$ \\
& 0.56 & 99.5 & $1.85 \times 10^{5}$ \\
Seawater & 10.56 & 94.6 & $1.75 \times 10^{4}$ \\
& 1.11 & 97.0 & $3.27 \times 10^{4}$
\end{tabular}



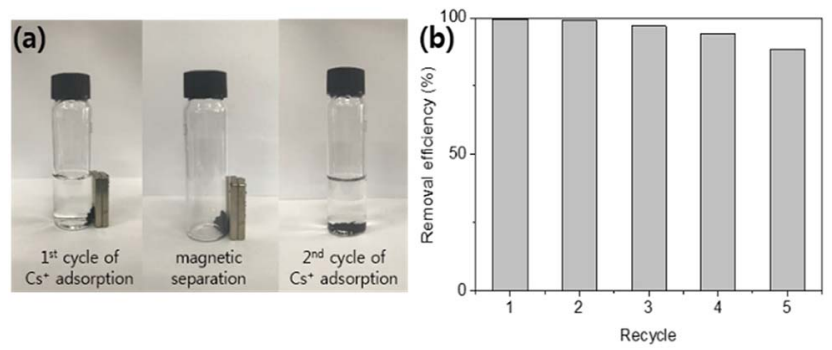

Fig. 8 (a) Images of cyclic $\mathrm{Cs}^{+}$adsorption and magnetic separation of the HMC. (b) Removal efficiency (\%) of the HMC in seawater (1-5 cycles)

the spent HMC after the water purification was totally isolated by magnetic separation, leading to the prevention of the generation of secondary wastes.

3.3.7 Adsorption mechanism. The $\mathrm{Cs}^{+}$ion exchange mechanism of KCuHCF in the HMC structure was elucidated using XPS and XRD analyses for the pristine HMC and $\mathrm{Cs}^{+}$ adsorbed HMC (200 mL of $1000 \mathrm{mg} \mathrm{L}^{-1} \mathrm{Cs}^{+}$solution; $m / V=1 \mathrm{~g}$ $\mathrm{L}^{-1}$ ) (Fig. 9). The full range spectra of the XPS (Fig. 9(a)) show the appearance of intense $\mathrm{Cs}^{+}$related peaks but do not show the presence of $\mathrm{K}^{+}$related peaks after the adsorption. In Fig. 9(b), the K2p XPS spectrum shows two main peaks at $291.83\left(2 \mathrm{p}^{3 / 2}\right)$ and $294.66 \mathrm{eV}\left(2 \mathrm{p}^{1 / 2}\right)$. After $\mathrm{Cs}^{+}$adsorption, the intensity of these two peaks significantly decreased. In the deconvolution of the Cs peaks (Fig. 9(c)), $3 \mathrm{~d}^{5 / 2}$ and $3 \mathrm{~d}^{3 / 2}$ at 724.22 and $738.15 \mathrm{eV}$, respectively, are clearly identified. Furthermore, the XRD peaks around $18^{\circ}, 40^{\circ}$, and $55^{\circ}$ disappeared following the $\mathrm{Cs}^{+}$ adsorption, and the remaining peaks correspond to the XRD pattern of CsCuHCF (PDF no. 24-0248) as seen in Fig. 9(d). It can be concluded that the mechanism of $\mathrm{Cs}^{+}$adsorption onto KCuHCF was accomplished by the exchange between $\mathrm{K}^{+}$and $\mathrm{Cs}^{+}$.
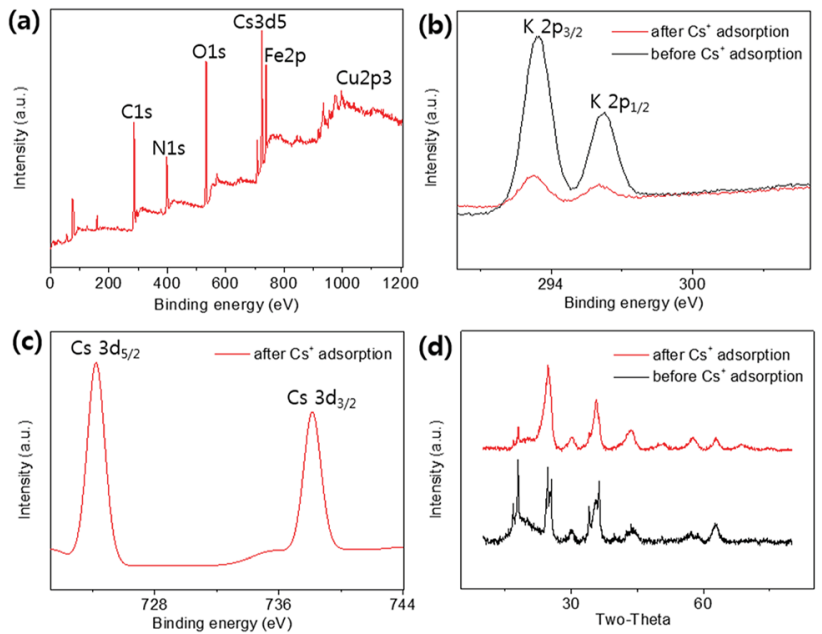

Fig. 9 (a) Full range XPS spectrum of the $\mathrm{HMC}$ after $\mathrm{Cs}^{+}$adsorption, and (b) the K $2 \mathrm{p}$ and (c) Cs 3d XPS spectra. (d) XRD patterns before and after $\mathrm{Cs}^{+}$adsorption of the HMC.

\section{Conclusions}

KCuHCF nanoparticles were incorporated into magnetic chitosan beads by coordination between amino groups and copper ions to remove $\mathrm{Cs}^{+}$from contaminated aqueous solutions. The grafting of amino groups to magnetic chitosan beads and the subsequent immobilization of a large quantity of KCuHCF nanoparticles on the HMC were confirmed in the FT-IR and SEM-EDS spectra, respectively. The $\mathrm{Cs}^{+}$adsorption performance of the HMC was evaluated regarding the $\mathrm{pH}$ stability, capacity, kinetics, selectivity, and reusability. In a broad range of $\mathrm{pHs}$ from 4 to 10, the HMC showed a stable removal efficiency above 94\%, and the $\mathrm{Cs}^{+}$ions were quickly removed from the aqueous solution (8.99 $\mathrm{mg} \mathrm{L}^{-1}, m / V=1 \mathrm{~g} \mathrm{~L}^{-1}$ ) within $4 \mathrm{~h}$. The maximum adsorption capacity was $136.47 \mathrm{mg} \mathrm{g}^{-1}$, which is one of the highest $\mathrm{Cs}^{+}$capacities among the previous reported magnetic $\mathrm{Cs}^{+}$adsorbents. In addition, the HMC could remove $\mathrm{Cs}^{+}$selectively (>97\%) from seawater $\left(1.11 \mathrm{mg} \mathrm{L}^{-1}\right)$, and this result is related to the well-dispersed KCuHCF nanoparticles in the HMC. Furthermore, the repetitive use of the HMC was possible with highly selective $\mathrm{Cs}^{+}$removal in seawater, and the $\mathrm{Cs}^{+}$ adsorbed HMC could be quickly recovered by magnets. This is important in terms of industrial applications to purify a large volume of contaminated water without secondary contamination. The comparison results of XPS and XRD between the pristine and $\mathrm{Cs}^{+}$exchanged HMC showed that this effective $\mathrm{Cs}^{+}$ removal performance was due to the ion exchange mechanism between the $\mathrm{K}^{+}$in the immobilized $\mathrm{KCuHCF}$ and the $\mathrm{Cs}^{+}$from the aqueous phase. Therefore, the HMC is one of the most favorable $\mathrm{Cs}^{+}$adsorbents with respect to not only economic cost and eco-friendly preparation but also its rapid and selective recovery of $\mathrm{Cs}^{+}$from an aqueous solution.

\section{Conflicts of interest}

There are no conflicts to declare.

\section{Acknowledgements}

This work was supported by the UK-Korea Joint Research Program through NRF grants (NRF-2015M2A7A1000219) and the Advanced Biomass R\&D Center as the Global Frontier Project (ABC-2010-0029728) funded by the Ministry of Science, ICT and Future Planning, South Korea. D. Harbottle acknowledges the support of Engineering and Physical Sciences Research Council grant number EP/M026426/1.

\section{References}

1 Y.-X. Wang, J.-R. Li, J.-C. E. Yang, B. Yuan and M.-L. Fu, RSC Adv., 2015, 5, 91431-91435.

2 International Energy Agency, Key World Energy Statistics, http:/www.iea.org/publications/freepublications/ publication/KeyWorld2017.pdf.

3 S. Baik, H. Zhang, Y. K. Kim, D. Harbottle and J. W. Lee, RSC Adv., 2017, 7, 54546-54553. 
4 H. Zhang, S. Tangparitkul, B. Hendry, J. Harper, Y. Kon Kim, T. N. Hunter, J. W. Lee and D. Harbottle, Chem. Eng. J., 2019, 355, 797-804.

5 P. J. Faustino, Y. Yang, J. J. Progar, C. R. Brownell, N. Sadrieh, J. C. May, E. Leutzinger, D. A. Place, E. P. Duffy, F. Houn, S. A. Loewke, V. J. Mecozzi, C. D. Ellison, M. A. Khan, A. S. Hussain and R. C. Lyon, J. Pharm. Biomed. Anal., 2008, 47, 114-125.

6 E. Calabrese, Proc. Natl. Acad. Sci. U. S. A., 2011, 108, 1944719448.

7 A. Nilchi, R. Saberi, M. Moradi, H. Azizpour and R. Zarghami, Chem. Eng. J., 2011, 172, 572-580.

8 Y. K. Kim, J. H. Park and J. W. Lee, Carbon, 2018, 126, 215224.

9 H. Yang, M. Luo, L. Luo, H. Wang, D. Hu, J. Lin, X. Wang, Y. Wang, S. Wang, X. Bu, P. Feng and T. Wu, Chem. Mater., 2016, 28, 8774-8780.

10 D. Alby, C. Charnay, M. Heran, B. Prelot and J. Zajac, J. Hazard. Mater., 2018, 344, 511-530.

11 Y. Kim, Y. K. Kim, J. H. Kim, M.-S. Yim, D. Harbottle and J. W. Lee, Appl. Surf. Sci., 2018, 450, 404-412.

12 E. Rathore, P. Pal and K. Biswas, Chemistry, 2017, 23, 1108511092.

13 Lalhmunsiama, C. Lalhriatpuia, D. Tiwari and S.-M. Lee, Appl. Surf. Sci., 2014, 321, 275-282.

14 T. Vincent, C. Vincent, Y. Barré, Y. Guari, G. Le Saout and E. Guibal, J. Mater. Chem. A, 2014, 2, 10007-10021.

15 T. Vincent, C. Vincent and E. Guibal, Molecules, 2015, 20, 20582-20613.

16 Y. Kim, Y. K. Kim, S. Kim, D. Harbottle and J. W. Lee, Chem. Eng. J., 2017, 313, 1042-1050.

17 Y. Zong, Y. Zhang, X. Lin, D. Ye, D. Qiao and S. Zeng, RSC Adv., 2017, 7, 31352-31364.

18 Y. K. Kim, T. Kim, Y. Kim, D. Harbottle and J. W. Lee, J. Hazard. Mater., 2017, 340, 130-139.

19 Y. K. Kim, Y. Kim, S. Kim, D. Harbottle and J. W. Lee, J. Environ. Chem. Eng., 2017, 5, 975-986.

20 Y. K. Kim, K. Bae, Y. Kim, D. Harbottle and J. W. Lee, J. Ind. Eng. Chem., 2018, 68, 48-56.

21 J. Y. Yoon, H. Zhang, Y. K. Kim, D. Harbottle and J. W. Lee, J. Environ. Chem. Eng., 2019, 7, 102824.

22 K.-M. Lee, T. Kawamoto, K. Minami, A. Takahashi, D. Parajuli, G. Kido, K. Yoshino and H. Tanaka, $R S C A d v$, 2016, 6, 16234-16238.

23 B. Folch, J. Larionova, Y. Guari, K. Molvinger, C. Luna, C. Sangregorio, C. Innocenti, A. Caneschi and C. Guerin, Phys. Chem. Chem. Phys., 2010, 12, 12760-12770.

24 D. Dechojarassri, S. Asaina, S. Omote, K. Nishida, T. Furuike and H. Tamura, Int. J. Biol. Macromol., 2017, 104, 1509-1516.

25 R. Wang, S. Gao, Z. Yang, Y. Li, W. Chen, B. Wu and W. Wu, Adv. Mater., 2018, 30.

26 E. Szymanska and K. Winnicka, Mar. Drugs, 2015, 13, 18191846.

27 S. Yang, D. Shao, X. Wang, G. Hou, M. Nagatsu, X. Tan, X. Ren and J. Yu, Mar. Drugs, 2015, 13, 3116-3131.

28 W. Jiang, W. Wang, B. Pan, Q. Zhang, W. Zhang and L. Lv, ACS Appl. Mater. Interfaces, 2014, 6, 3421-3426.
29 S. Yang, N. Okada and M. Nagatsu, J. Hazard. Mater., 2016, 301, 8-16.

30 P. S. Barber, S. P. Kelley, C. S. Griggs, S. Wallace and R. D. Rogers, Green Chem., 2014, 16, 1828-1836.

31 S. Chatterjee, M. W. Lee and S. H. Woo, Bioresour. Technol., 2010, 101, 1800-1806.

32 J. Duan, X. Liang, Y. Cao, S. Wang and L. Zhang, Macromolecules, 2015, 48, 2706-2714.

33 L. Lv, N. Chen, C. Feng, Y. Gao and M. Li, J. Taiwan Inst. Chem. Eng., 2017, 78, 485-492.

34 M. K. Sureshkumar, D. Das, M. B. Mallia and P. C. Gupta, J. Hazard. Mater., 2010, 184, 65-72.

35 W. Zhang, S. Jia, Q. Wu, S. Wu, J. Ran, Y. Liu and J. Hou, Mater. Sci. Eng., C, 2012, 32, 381-384.

36 A. Mohseni-Bandpi, B. Kakavandi, R. R. Kalantary, A. Azari and A. Keramati, RSC Adv., 2015, 5, 73279-73289.

37 S. Naeimi and H. Faghihian, Sep. Purif. Technol., 2017, 175, 255-265.

38 S. Zavareh, Z. Behrouzi and A. Avanes, Int. J. Biol. Macromol., 2017, 101, 40-50.

39 M. Rajiv Gandhi and S. Meenakshi, Carbohydr. Polym., 2013, 91, 631-637.

40 H. Li, S. Bi, L. Liu, W. Dong and X. Wang, Desalination, 2011, 278, 397-404.

41 B. D. Mather, K. Viswanathan, K. M. Miller and T. E. Long, Prog. Polym. Sci., 2006, 31, 487-531.

42 E. S. Read, K. L. Thompson and S. P. Armes, Polym. Chem., 2010, 1, 221-230.

43 C. Zhang, H. Zhang, R. Li and Y. Xing, RSC Adv., 2017, 7, 48189-48198.

44 H. Zhang, Q. Dang, C. Liu, D. Cha, Z. Yu, W. Zhu and B. Fan, ACS Appl. Mater. Interfaces, 2017, 9, 11144-11155.

45 Z. Zhou, F. Jiang, T.-C. Lee and T. Yue, J. Alloys Compd., 2013, 581, 843-848.

46 C. Cao, L. Xiao, C. Chen, X. Shi, Q. Cao and L. Gao, Powder Technol., 2014, 260, 90-97.

47 C. Dwivedi, S. K. Pathak, M. Kumar, S. C. Tripathi and P. N. Bajaj, Environ. Sci.: Water Res. Technol., 2015, 1, 153160.

48 A. Anuradha, S. Kumari, S. Layek and D. D. Pathak, New J. Chem., 2017, 41, 5595-5604.

49 H.-M. Yang, C. W. Park, P. K. Bae, T. Ahn, B.-K. Seo, B. H. Chung and J.-D. Kim, J. Mater. Chem. B, 2013, 1, 3035-3043.

50 C. Dong, W. Chen, C. Liu, Y. Liu and H. Liu, Colloids Surf., A, 2014, 446, 179-189.

51 M. E. A. Ali, F. M. Hassan and X. Feng, J. Mater. Chem. A, 2016, 4, 6620-6629.

52 H. Yang, H. Li, J. Zhai, L. Sun, Y. Zhao and H. Yu, Chem. Eng. J., 2014, 246, 10-19.

53 H. Yang, L. Sun, J. Zhai, H. Li, Y. Zhao and H. Yu, J. Mater. Chem. A, 2014, 2, 326-332.

54 K. S. Hwang, C. W. Park, K.-W. Lee, S.-J. Park and H.-M. Yang, Colloids Surf., A, 2017, 516, 375-382.

55 H. Zhang, X. Zhao, J. Wei and F. Li, Nucl. Eng. Des., 2014, 275, 322-328.

56 Y.-W. Chen and J.-L. Wang, Nucl. Sci. Tech., 2016, 27, 43. 
57 C. Thammawong, P. Opaprakasit, P. Tangboriboonrat and P. Sreearunothai, J. Nanopart. Res., 2013, 15, 1689.

58 Y. Zhu, W. Wang, H. Zhang, X. Ye, Z. Wu and A. Wang, Chem. Eng. J., 2017, 327, 982-991.
59 J. L. Mertz, Z. H. Fard, C. D. Malliakas, M. J. Manos and M. G. Kanatzidis, Chem. Mater., 2013, 25, 2116-2127. 